Voix et Images

volxetimages

\title{
Sémiotique et poétique « Les Chats » d'Émile Nelligan
}

\section{Guy Laflèche}

Volume 4, numéro 1, septembre 1978

Rina Lasnier

URI : https://id.erudit.org/iderudit/200136ar

DOI : https://doi.org/10.7202/200136ar

Aller au sommaire du numéro

\section{Éditeur(s)}

Les Presses de l'Université du Québec

\section{ISSN}

0318-9201 (imprimé)

1705-933X (numérique)

Découvrir la revue

Citer cet article

Laflèche, G. (1978). Sémiotique et poétique « Les Chats » d'Émile Nelligan. Voix et Images, 4(1), 50-76. https://doi.org/10.7202/200136ar d'utilisation que vous pouvez consulter en ligne.

https://apropos.erudit.org/fr/usagers/politique-dutilisation/ 


\title{
Sémiotique et poétique «Les Chats» d'Émile Nelligan
}

\author{
Un chat est un chat, une pomme \\ une pomme; mais un texte n'est pas \\ un texte.
}

G.-André Vachon

Sémiotique et poétique, les belles et chères mamelles de la critique universitaire actuelle : quel profil! Mon titre vous dessine une de ces louves a faire crever d'envie nos trois ou quatre revues savantes toutes prêtes à se prendre comme Voix et images pour des «instruments de références indispensables". Mais mon titre seulement, et pour une raison toute simple : avec un petit effort, je crois qu'il ne sera pas tout à fait impossible de lire le texte qu'il coiffe. En effet, rien n'est plus amusant que d'écrire des textes dont on se dispenserait bien. Voici donc un exercice d'analyse littéraire où je me suis amusé à mettre la sémiotique à sa place - et le plus sérieusement du monde: au sens strict (voir la figure 1). Car le plus souvent, le sémioticien prend d'abord une grande inspiration, vous comprenez qu'il va faire de la sémiotique, puis vous ne comprenez plus rien. Si l'on admet que la sémiotique est une science de la signification, on peut penser qu'elle ne devrait pas en être dépourvue. Je me suis donc efforcé de définir et d'illustrer la signification de cette science de la signification dans le champ des études littéraires, et sur un exemple particulier.

Comme les sémioticiens sont les derniers venus dans le monde trouble des études littéraires, on a tendance à les placer au sommet et à l'avant-garde: or il est plaisant de voir en pratique que leur place, pourtant enviable, est à l'entresol. Et cette illusion de l'avant-garde me semble pleine d'enseignement: elle montre que la transposition mythique qui fait passer le progrès scientifique du temps à l'espace est communément représentée par un saut ou une avancée, tandis qu'en réalité le véritable progrès scientifique n'est jamais une addition, mais toujours une révolution, c'est-à-dire une réorganisation de notre univers mental. Ainsi la sémiotique littéraire ne décrit pas la fin du texte littéraire, sa valeur poétique, celle que déploie rigoureusement la critique thématique (comme 
toujours, mais sans rigueur, la première glose venue) : elle décrit quelque chose de beaucoup plus simple (et c'est en cela que réside la difficulté), soit les moyens qu'a le texte pour parvenir à cette fin ou de se faire littéraire. Autrement dit, la tâche difficile de la sémiotique est simplement de décrire la transformation de la langue en langage et, dans le cas particulier de la sémiotique littéraire, de dire comment ou à quelles conditions la langue devient un langage poétique. Bref, elle doit montrer comment on peut passer du plan stylistique au plan poétique d'un texte.

Voilà l'essentiel de ce que j'ai tenté d'illustrer ici. Je situerai d'abord la sémiotique littéraire parmi les autres sciences de la littérature, puis je décrirai brièvement "les Chats" d'Émile Nelligan à partir des trois grandes sciences fondamentales du texte et en particulier du point de vue de la sémiotique (que j'aurai définie comme al'étude du traitement de la signification dénotée $"$ ) que je confronterai aux deux autres. Le poème intitulé «les Chats» de Nelligan constituera donc le champ d'application et de confrontation des quelques présupposés théoriques que j'introduirai; j'en recopie tout de suite le texte que je désignerai ensuite par le sigle $\mathrm{CdN}$ :

\section{LES CHATS}

Aux becs de gaz éteints, la nuit, en la maison, Ils prolongent souvent des plaintes éternelles;

Et sans que nous puissions dans leurs glauques prunelles

En sonder la sinistre et mystique raison.

Parfois, leur dos aussi secoue un long frisson;

Leur poil vif se hérisse à des jets d'étincelles

Vers les minuits affreux d'horloges solennelles

Qu'ils écoutent sonner de bizarre façon.

Remarque sur l'édition des $\mathrm{CdN}$ : Ce texte a été édité pour la première fois par Luc Lacourcière dans les Poésies complètes d'Emile Nelligan, Montréal, Fides, 1952, $2^{e}$ éd., 1958, p. 269. Comme l'explique l'éditeur, ce poème fait partie de la collection Nelligan-Corbeil (actuellement à la Bibliothèque nationale du Québec) parmi 24 poèmes dont 19 étaient inédits. II n'a donc jamais été publié par Nelligan; ni par son premier éditeur, Louis Dantin, parce que "l'ensemble lui a paru inférieur" (ibid., p. 316). Voir aussi Paul Wyczynski, Bibliographie descriptive d'Emile Nelligan, Ottawa, Ed. de I'Université d'Ottawa, 1973, p. 28.

\section{Remarques}

1. Le texte qu'on commence à lire constitue la version écrite de l'exposé que je devais faire le 25 octobre 1976 et qui a été annulé à cause de la grève des professeurs. Cet exposé était annoncé sous le titre "Sémiotique et poétique" et devait constituer l'une des cinq séances du séminaire du programme de maîtrise appelé Problèmes de sémiotique (U.Q.A.M., Département de philosophie, automne 1976). Ce travail m'avait été demandé par le responsable de ce séminaire, M. André Vidricaire. J'avais déjà achevé l'essentiel de ce travail d'analyse lorsque j'ai lu pour la première fois en septembre 1976 le titre de mon exposé, titre qui lui donne un caractère à la fois et contradictoirement "populaire", "original " et "difficilen: ce titre tend à placer ce travail particulier, comme l'ensemble de mon travail, à la pointe de la recherche, non seulement dans le domaine des études littéraires, mais également dans les sciences de l'homme où les problèmes de la communi- 
cation, de l'information et de la signification occupent actuellement une position qu'on peut bien dire privilégiée. C'est en l'acceptant au pied de la lettre et avec raison (en particulier en consacrant mon introduction à la situation et à la définition des mots qu'on y trouve) que j'entends ne pas être dupe du titre que je donne maintenant à ce travail.

2. J'ai déjà utilisé plusieurs fois le mot «travail» dans la remarque précédente: on aura donc compris que je maintiens absolument ici les présupposés théoriques que j'ai établis explicitement en tête de mon Petit Manuel des études littéraires pour une science générale de la littérature paru chez VLB Éditeur en 1977. Aucun de ces présupposés ne sera "démontré" dans ce texte: ils seront simplement utilisés, je l'espère, avec évidence. Je précise seulement que les études littéraires sont un ensemble d'activités dont le produit s'appelle la connaissance, ce qui est le cas de toutes les sciences; dans le cas des études littéraires, il s'agit plus particulièrement d'une description des textes littéraires et de la littérature. De façon plus particulière encore, mon travail a consisté ici à décrire les $\mathrm{CdN}$ : je ne me propose donc pas de faire une “explication de texte" mais, précisément, une udescription des $\mathrm{CdN}$ ». J'ajoute que je n'ai pas encore terminé ce travail, de sorte que ce texte doit être considéré comme la première partie de ce qui deviendra, si j'en ai un jour l'occasion, une étude des CaN. Bien entendu, on peut croire qu'il s'agit d'une précaution destinée à parer à la critique, mais je voudrais aussi qu'on voie dans cette réserve l'intention de présenter un état et non le résultat de mon travail de recherche.

3. Est-ce que le texte de Nelligan qui sera décrit ici est un bon poème? J'avoue en toute candeur que je ne m'étais jamais posé la question avant qu'on ne me dise qu'en vérité je faisais une belle étude [merci] d'un poème médiocre. Pourtant, beau ou laid, bien ou mal écrit, d'une grande valeur ou d'une valeur médiocre, ces jugements ne sauraient décrire que ma relation au poème de Nelligan et ne disent rien du texte. Par conséquent, si je dis que j'aime ce poème, cela peut intéresser mon psychanalyste et les voyeurs, mais personne d'autre. Bref, il s'agit d'une question sans intérêt à laquelle la critique policière s'accroche afin de parler d'elle sans trop se compromettre - puisque dans tous les cas elle en laissera la responsabilité à Nelligan qui l'aura bien ou mal écrit.

4. Quel est alors l'intérêt de la description? Nul, si le lecteur s'intéresse à moi ou à lui-même. Si je veux connaître les raisons de mes amours, je n'ai d'autres ressources que l'analyse (et aucune raison d'en faire des textes de «critique littéraire»). Voilà pourquoi je répète qu'on ne trouvera ici qu'un exercice d'analyse littéraire, à savoir une inutile description du poème de Nelligan. Car sauf à (faire) croire que le Savoir est le résultat d'une Révélation, il faut maintenir absolument le postulat matérialiste que j'ai essayé de pratiquer dans cette étude : la description d'un texte littéraire représente notre savoir actuel sur lui. Or la connaissance, qui est un plaisir absolu de l'esprit, ne saurait être que le résultat d'un travail, comme toute production, et le travail est alors un plaisir - du moins chaque fois que la force de travail n'est pas transformée en ces besognes policières qui consisteraient ici, par exemple, à sacraliser le poème ou la poésie de Nelligan au profit d'impératifs retors et moraux : la beauté, la bonté et la valeur des textes de Nelligan, ou leur mise à l'index.

5. En conséquence, le travail qui suit n'est et ne devrait être que la description d'un poème de Nelligan. Si je veux préciser qu'il s'agit d'une description scientifique, ce sera surtout pour dire que je l'ai voulue la plus simple et la plus efficace possible. Et si elle peut paraître parfois difficile, ce n'est pas parce qu'il y est 
question de sémiotique qui n'est pas une science plus "difficile " que la stylistique ou la poétique; ce sera simplement parce qu'en voulant être complet et explicite, je n'ai peut-être pas réussi à trouver partout la solution la plus élégante. Dans ce cas, c'est en complétant, en corrigeant et en simplifiant mon travail que le lecteur aura autant de plaisir que j'en ai eu à le réaliser. J'ajoute enfin que je ne crois pas que ce texte descriptif soit bien écrit et que je ne l'ai pas voulu ainsi : je n'ai cherché qu'à décrire simplement et rigoureusement l'état actuel de mon travail. Bref, il ne s'agit pas d'un texte littéraire, mais d'un simple rapport de recherche.

\section{Introduction : Stylistique, sémiotique et poétique}

Le premier mot du titre de ce texte, repris du nom du séminaire où il devait figurer, est "sémiotique". Je peux essayer de le démystifier simplement, comme le fait le dictionnaire courant (le Petit Robert, par exemple, mais qui en fait un équivalent de "sémiologie"), en le plaçant dans divers contextes. Soit l'avalanche des définitions suivantes que je propose d'adopter.

\subsection{La sémiologie}

La sémiologie, prophétisée par Saussure/Peirce, structurée par Luis Prieto et largement vulgarisée et diffusée par Georges Mounin, est l'étude scientifique des systèmes de signes, c'est-à-dire des codes. Les signes sont des indices humains artificiels construits pour contenir, transporter et communiquer de l'information: ils constituent ce qu'on doit nommer, au sens strict, des messages.

Exemples de codes: le code de la route, la langue française, le langage de programmation FORTRAN, les règles de l'étiquette.

Bibliographie sommaire :

Ferdinand de Saussure, Cours de linguistique générale, $3^{\theta}$ éd., Paris, Payot, 1967 (éd. critique de Tullio de Mauro, 1973), p. 33, 35, 100-101.

Luis J. Prieto, "la Sémiologie" dans le Langage, Encyclopédie de la

Pléiade, 1968, p. 93-144 ; Messages et Signaux, P.U.F., 1966, 1972.

Georges Mounin, Introduction à la sémiologie, Paris, Éd. de Minuit, 1970.

Roland Barthes, Éléments de sémiologie, à la suite du Degré zéro de l'écriture, Paris, Gonthier, 1969.

On doit remarquer que le signe se distingue de l'indice en ce qu'il est artificiel : il doit être décodé, tandis que l'indice est interprété, ce qui permet d'opposer les sciences dites exactes qui interprètent les indices (" naturels »), les sciences de l'homme qui étudient les indices humains et, parmi celles-ci, la sémiologie qui étudie les indices humains artificiels, soit les signes proprement dits et les systèmes de signes.

Dans l'exacte mesure où les arts peuvent être considérés comme des systèmes de communication, il existe une sémiologie de chacun d'entre eux: une sémiologie du cinéma, de la littérature, de la musique, etc., ce qui repose chaque fois sur le postulat que quelque chose est communiqué par un destinateur à un destinataire, de sorte qu'on peut se proposer d'étudier les messages de Giacometti, de Céline, de Stravinski, de Fellini, etc. Bien entendu, ce postulat n'est pas encore démontré - ni 
infirmé : on ne sait pas si les arts sont ou non des sytèmes de communication, ni dans quelle mesure. C'est pourtant l'utilisation de ce postulat à titre d'hypothèse qui a conduit à la confusion et à la distinction avec ce dont il sera question maintenant, la sémiotique. Remarquons d'abord que les systèmes de communication et les signes qui les constituent peuvent également être considérés comme des indices : s'ils sont créés pour être décodés, ils peuvent toujours être aussi interprétés - ce qui sera pour une part l'objet de la sémiotique.

\subsection{La sémiotique}

La sémiotique qui est également pratiquée sous le nom de «sémiologie " et parfois sous son nom - ce qui est le cas en particulier pour la sémiotique littéraire -, mais qui n'a jamais encore été structurée, est l'étude scientifique de la signification des systèmes de communication: ou bien l'étude du traitement de la signification par les différents systèmes de signes, ou bien encore l'étude de la signification de ces systèmes de signes eux-mêmes.

Dans un cas particulier, celui du traitement de la signification par le langage humain articulé (soit la langue qu'étudie la linguistique), la sémiotique est actuellement en voie de constitution : c'est la sémantique. Partout ailleurs, et notamment dans le cas de tous les arts - et donc de la littérature - la sémiotique devra réussir à se constituer probablement comme une partie de la sémiologie, exactement comme la sémantique constitue une partie de la linguistique. On voit tout de suite que dans le cas de la sémiotique littéraire, cette science risque de se noyer dans les nombreuses pratiques de la “critique" (c'est-à-dire les "lectures" aussi nombreuses que les «lecteurs" qu'on appelle les «critiques") ou d'être confondue avec ce qu'elle devra largement appliquer, la sémantique, elle-même fortement redevable des méthodes et des procédures de la linguistique. C'est pourtant cette deuxième voie qu'il faut choisir, attendu que la langue est à la littérature ce que la sémantique est à la sémiotique : son matériau.

Remarque : dans cette dernière phrase, comme toujours à partir d'ici, j'entends par "sémiotique" la "sémiotique littéraire". Ce sera à une sémiotique gênérale de rationaliser les présupposés de l'étude (des systèmes) de la signification des systèmes de communication telle que je me contente de la supposer ici.

Bibliographie sommaire:

Bernard Pottier, "Vers une sémantique moderne" dans les Travaux de linguistique et de littérature, Strasbourg, 1964, vol. 2, p. 107-132.

A. J. Greimas, Sémantique structurale, Paris, Larousse, 1966.

(Remarque: du premier au second texte, on assiste au passage de la sémantique à la sémiotique. Les travaux les plus connus de la sémiotique littéraire appartiennent d'ailleurs à Greimas et à son école; par exemple: A. J. Greimas, Du sens, Essais sémiotiques, Paris, Seuil, 1970; Jean-Claude Coquet, Sémiotique litteraire, Paris, Mame, "Univers sémiotiques", 1972; François Rastier, Essais de sémiotique discursive, Paris, Mame, "Univers sémiotiques $», 1973$.) 
Je précise toutefois qu'à mon sens la sémiotique est la science de la littérature dont l'objet est la description du contenu immédiat du texte littéraire, c'est-à-dire l'étude du traitement de sa signification dénotée.

\subsection{La poétique}

Le titre de ce texte se contente de confronter la sémiotique à la poétique, mais alors que la sémiotique étudie la signification des systèmes de communication, la poétique étudie la signification d'un système de communication particulier: celui du langage poétique. Je suis fermement convaincu que ce système de communication constitue l'essentiel de tous les arts, mais je crois avoir déjà illustré qu'il s'agit au moins d'un système essentiel à l'un d'entre eux: la littérature. Le texte littéraire se définit, je pense, en ce qu'il articule toujours une communication poétique qui est connotée par ce qu'on a toujours nommé la rhétorique et dont le contenu n'est rien d'autre que ce qu'on a appelé depuis quelques dizaines d'années la thématique (ou plutôt la "critique thématique").

Remarque: sur l'étude de ce mécanisme, je me permets de renvoyer d'abord à ma thèse, Mallarmé, Grammaire générative des "Contes indiens", Montréal, Les Presses de I'Université de Montréal, 1975; et surtout au bref article suivant: "Les Règles de réécriture mallarméenne des Contes indiens " dans Analyse du discours, P.R. Léon et H. Mitterand (édit.), Montréal, Le Centre éducatif et culturel, 1976.

Cette connotation proprement littéraire (car il existe aussi plusieurs connotations translittéraires qu'étudient par exemple la psychocritique et la sociocritique) n'est donc qu'un cas particulier de la sémiologie de la littérature (la rhétorique comme système de communication) de même que de la sémiotique littéraire (la thématique comme système de signification) auxquelles on réservera le nom de poétique (littéraire).

Enfin, il faut faire attention de ne pas confondre un genre littéraire particulier, celui que l'on nomme la poésie, ou les poèmes qui le constituent, avec le langage que la poétique étudie: tous les textes littéraires sont, par définition, "poétiques", mais dans le cas du poème la poétique apparaît souvent comme le seul plan de la signification, de sorte que se trouve posé avec acuité le problème du plan de la sémiotique.

\subsection{La stylisitique}

La stylistique qui, avec la sémiotique et la poétique, est la troisième des grandes sciences du texte littéraire constitue l'absence remarquable du titre de ce texte. II faut dire que depuis quinze ans la stylistique a été l'objet d'une crise qui l'a rendue bien suspecte, à ce point qu'on peut se demander si cette science millénaire n'est pas disparue au profit des si populaires "sémiotiques" et "poétique". C'est en 1959 que Gérald Antoine fait la synthèse critique remarquable des travaux de Charles Bally et de Leo Spitzer qui représentent respectivement la doctrine stylistique française telle qu'elle est consignée dans les manuels depuis le début du siecle et ce qu'on peut appeler la «critique stylistique» qui s'est 
toujours pratiquée sans doctrine, comme toute «critique littéraire». Or cette même année marque le début de la dislocation de cette science sous l'impact de ce qu'on appelle alors laulinguistique structurale": la stylistique va dès ce moment se déployer sur trois axes :

a) L'étude des systèmes linguistico-littéraires: soit par exemple les systèmes d'élocution narratifs (histoire/discours) dégagés par Émile Benveniste en 1959, ou encore la description du système du langage poétique élaborée par Roman Jakobson l'année suivante sous le nom de "fonction poétique"; ce dernier exemple a d'ailleurs la valeur d'un indice déterminant puisqu'il donnera naissance à une deuxième voie de la stylistique :

b) L'étude de la rhétorique ou plutôt la reformulation de la rhétorique classique à la lumière de la linguistique structurale et l'application de cette néo-rhétorique à l'analyse stylistique: soit le livre de Jean Cohen sur la Structure du langage poétique (Flammarion, 1966) et la Rhétorique générale du groupe de Liège (Larousse, 1970) qui le développe.

c) L'étude du texte littéraire comme "totalité en fonctionnement» dont la première illustration, magistrale, me paraît être constituée par l'étude des "Chats" de Baudelaire par Roman Jakobson et Claude Lévi-Strauss (1962) qui a été reprise partout dans d'innombrables analyses depuis lors et dont on trouve une brève synthèse dans le livre de Daniel Delas et Jacques Filliolet (Larousse, 1973).

Remarque: Je renonce à produire une autre esquisse bibliographique que celle qu'on pourra reconstituer facilement à l'aide du dernier paragraphe, d'autant que les textes auxquels je viens de faire allusion sont tous très connus. Je précise seulement que "les Chats de Charles Baudelaire" est d'abord paru dans l'Homme, janvier-avril 1962, vol. II, $n^{\circ}$ 1, p. 5-21.

Sur chacune de ces trois voies, la stylistique a mis en cuvre un dénominateur commun remarquable: l'utilisation rigoureuse des procédures et des concepts de la linguistique appliqués aux textes littéraires (“les Chats" de Baudelaire) ou à la littérature (soit les formes littéraires comme les types de discours narratifs); en cela, la stylistique peut être définie très simplement comme l'étude scientifique de l'expression du texte littéraire: il ne peut s'agir que d'un domaine particulier de la linguistique, soit l'étude de ce que les linguistes appellent l' "usage littéraire " de langue.

\subsection{Les trois plans du texte littéraire}

Voilà donc brièvement définis les trois grands plans du texte littéraire ou, ce qui revient au même, les trois grandes sciences à partir desquelles il peut être décrit. II reste à les situer clairement les unes par rapport aux autres. Soit le sous-titre de cet article :

"Les chats " d'Émile Nelligan

1. La stylistique étudie l'expression (le linguiste dit le «signifiant") de cette phrase: elle dira, par exemple, qu'il s'agit essentiellement 
d'une phrase nominale constituée de deux groupes qu'elle décrira comme des noms propres et dont l'un est subordonné à l'autre par un mot de relation, de sorte que le deuxième est complément (déterminatif) du premier.

2. La sémiotique étudie la signification transportée immédiatement par cette phrase (le linguiste dit le «contenu dénoté» et appelle cette science la "sémantique"): elle décrira, par exemple, ce qui est réellement désigné par chacun des deux noms propres et en particulier le texte (CdN) qu'on a déjà lu qui se trouve désigné par leur conjonction; elle pourrait encore étudier le rapport entre le sous-titre et le sujet du texte.

3. La poétique, enfin, étudie la signification poétique transportée par cette expression (le linguiste dit le "contenu connoté " et nous l'avons déjà appelé la thématique): elle étudiera l'allusion (disons: la connotation culturelle) à l'article de Jakobson et de Lévi-Strauss dont il a déjà été question, de même que le rappel ironique (que ces deux titres ont en commun) avec l'expression de Boileau devenu un adage populaire: «appeler un chat un chat».

\section{Remarques}

1. Bien entendu, le sous-titre qui vient de servir d'exemple n'est pas un texte littéraire et n'en est pas extrait : cela illustre bien que ni la stylistique, ni la sémiotique, ni la poétique ne se limitent à l'étude du texte littéraire; ces trois sciences fondamentales du texte s'appliquent même probablement à tous les arts.

2. On peut schématiser de la manière suivante les trois plans fondamentaux du texte littéraire en gardant à l'esprit que ces trois plans ne sont pas des "parties" du texte, mais bien des points de vue ou des sciences à partir desquels tout texte peut être décrit :

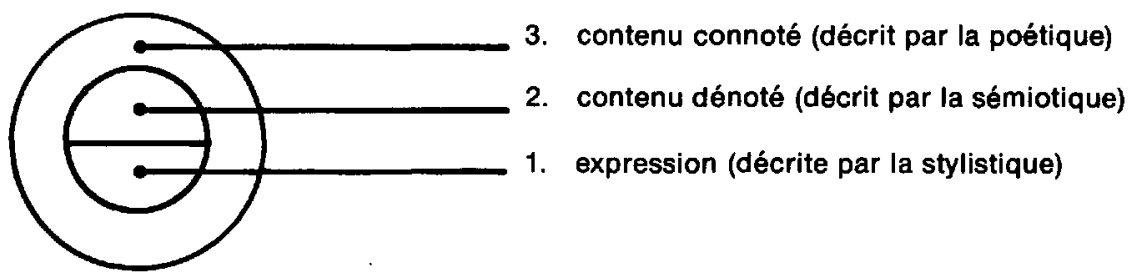

Figure 1. Représentation spatiale des trois plans du texte littéraire.

3. Si aucune de ces trois sciences n'occupe une position privilégiée par rapport aux deux autres, les trois plans du texte littéraire qu'elles décrivent sont au contraire hiérarchisés, et dans l'ordre des numéros de la figure 1: la stylistique étudie le plan de l'expression qui sert à dénoter le plan qu'étudie la sémiotique et à connoter avec lui le plan poétique qui constitue la fin du texte littéraire. La sémiotique n'occupe donc pas la position privilégiée que lui donne le titre de cet article et la vogue actuelle: d'abord parce qu'aucune science, comme activité de connaissance, ne peut prétendre à l'hégémonie: elle est, par définition, un point de vue parmi d'autres; ensuite parce que la sémiotique, si elle est bien la dernière née des sciences de la littérature, décrit un plan secondaire du texte, soit un moyen d'atteindre sa signification poétique qui constitue sa fin. Cette remarque ne constitue pas un jugement de valeur, mais la reconnaissance d'une antériorité logique: un texte doit dénoter une signification avant d'en connoter une. 


\section{Les CdN, un poème: Introduction à l'intertextualité}

Avant d'analyser les $\mathrm{CdN}$ à partir des trois points de vue dont il vient d'être question, c'est-à-dire avant de l'étudier comme un texte littéraire, il convient de décrire ce qui classe ce texte dès le premier coup d'œil et encore à la première lecture comme une actualisation particulière de la littérature, ou du moins de quelques-unes de ses formes. Je précise d'abord que la littérature est une institution qu'on ne connaît que par les textes littéraires qu'elle transcende toujours: on peut dire que la littérature n'existe jamais dans un texte, pas plus que dans des milliers, mais qu'elle existe et ne peut exister qu'entre deux ou des textes. En pratique, les $\mathrm{CdN}$ actualisent la littérature par les rapports que ce texte établit avec d'autres ou par les formes qu'il leur emprunte. Soit d'abord la forme versifiée ou la versification qui classe irrémédiablement les CdN dans le genre dénommé «poésie»: il s'agit de deux quatrains (strophes de quatre vers) d'alexandrins (vers de douze ou treize syllabes) construits sur deux rimes embrassées : une rime masculine en [j] appuyée par deux phonèmes

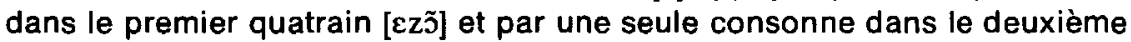

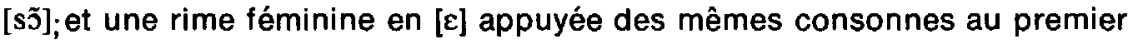
quatrain comme au septième vers [nعl(ə)], tandis qu'au sixième vers, seule la consonne finale appuie la rime $[\varepsilon l(\partial)]$ : on remarque donc que dans le deuxième quatrain toutes les rimes sont suffisantes tandis que dans le premier elles sont riches et qu'en plus ces deux rimes assonnent entre elles sur la voyelle $[\varepsilon]$ qui constitue la rime féminine et un phonème d'appui des rimes masculines.

Remarque: sur la terminologie employée ici pour décrire la versification se reporter a Maurice Grammont, Petit Traité de versification française, Paris, Armand Colin, «U», 1965, 1969.

On peut donc parler de l'unité harmonique du premier quatrain dans la mesure où tous ses vers riment ou assonnent et parce qu'on ne rencontre - et à la fin seulement - qu'une allitération ou qu'une série de répétitions de phonèmes, soit le bloc phonétique constitué par «sinistre et mystique" [sinistremistik] où le phonème [i] est répété quatre fois dont deux dans le même entourage [ist].

Au contraire, dans le deuxième quatrain, de telles allitérations se rencontrent à la fin de chaque vers : «long frisson " [lõfrisj̄] où la rime se trouve en écho; "des jets d'étincelles" [d $\left.\left.\varepsilon_{3} \varepsilon \operatorname{det} \tilde{s} s \varepsilon\right](a)\right]$ où la rime se trouve deux fois en écho et la dentale [d] répétée; "horloges solennelles" [orlozəsolencl(ə) ] où la rime est encore reprise en écho et la voyelle [ə] reprise trois fois; et "bizarre façon" [bizarəfasõ] où la voyelle [a] est répétée. Si ces allitérations marquent nettement les fins de vers (et compensent la rime suffisante), elles brisent l'unité harmonique du deuxième quatrain puisqu'elles sont arbitraires aussi bien du point de vue de la versification que de la phonétique, contrairement à la rime ou à l'assonnance qui compte la fin de vers. Cette brisure de l'harmonie du deuxième quatrain paraît d'autant plus remarquable qu'il s'ouvre sur deux hiatus: 
«dos aussi» [doosi] au cinquième vers, qui est le plus net en ce qu'il ne peut être lu que par un allongement de la voyelle [do:si], de même que "se hérisse» [sæeris(ə)] où l'hiatus est dû à l'impossibilité d'élider le pronom réfléchi devant ce qu'on appelle traditionnellement le $\mathrm{h}$ aspiré. Toutefois, ces deux hiatus et surtout les redondances phonétiques du deuxième quatrain ne sont pas assez marqués pour faire éclater ce qu'on peut continuer d'appeler l'unité harmonique des $\mathrm{CdN}$; d'autant plus que l'alexandrin connaît toujours dans ce poème une césure régulière après la sixième syllabe et qu'on n'y trouve aucune diérèse (semi-voyelle constituant à elle seule une syllabe). Bref, si ce n'était des hiatus, il n'y aurait rien d'autre à dire pour décrire la versification et la structure phonétique des $\mathrm{CdN}$ que ce qu'on a dit dès le début: il s'agit de deux quatrains d'alexandrins de deux rimes embrassées. Mais ce serait déjà assez, on le voit, pour le situer dans la poésie moderne d'expression française. Un coup d'cil sur sa rhétorique la plus explicite peut nous permettre de le situer, je pense, plus précisément dans le symbolisme romantique de la fin du siècle dernier et du début de ce siècle.

En dehors des structures phonétiques dont il vient d'être question, on ne trouve aucun élément lexical, morphologique ou syntaxique remarquable qui aurait pu, par exemple, être imposé par la versification: soit un mot rare, technique, savant, précieux ou étranger qui serait amené par la rime; on n'en trouve même aucun dans tout le poème.

Remarque: «Bec de gaz» est cette pièce de la lampe qui contrôle et distribue le débit du gaz qu'elle brûle; "glauque» est une teinte du vert, et «poil» est la catachrese (figure convenue, ici une 'synecdoque de la matiere) pour le pelage ou la fourrure. Tout le lexique des $\mathrm{CdN}$ est d'une banalite remarquable.

On peut seulement remarquer que les rimes masculines sont toutes constituées par des substantifs singuliers de deux syllabes («maison»/ «raison "/ "frisson "/ «façon"), tandis que les rimes féminines opposent dans chaque quatrain un substantif féminin pluriel ("prunelles» et «étincelles») à un adjectif qui constitue l'extension d'un substantif de même genre et de même nombre (“plaintes éternelles" et "horloges solennelles»); de sorte que la rime est très riche «pour l'œil » avec des rimes aussi catégorielles (c'est-à-dire appartenant aux mêmes catégories grammaticales). Après les niveaux lexical et morphologique, le niveau syntaxique présente le même caractère de régularité qui appuie l'unité harmonique des CdN : sauf le troisième vers, suivi par une extension du syntagme verbal (soit le groupe verbal qui complète le semi-auxiliaire et qui forme bien évidemment à ce titre une unité syntaxique), chaque vers se termine par un caractère de ponctuation (les vers $1,2,4,5$ et 8 ) ou commence, au contraire, par un mot de relation (les vers 3,6 et 7 ), et trois vers $(1,5$ et 8 ) accumulent les deux caractères. Du point de vue de la versification, on ne décrit pas autre chose lorsqu'on dit que les $\mathrm{CdN}$ ne connaissent ni enjambement, ni rejet, c'est-à-dire que les pauses métriques sont également des pauses syntaxiques. $A$ tous les niveaux linguistiques, on trouve donc 
ce qu'on peut appeler la simplicité de la versification romantique héritée de la poésie française classique: aucune des extravagances lexicales de la poésie baroque (même pas la plus petite allusion mythologique) et aucune des cassures de la versification qui caractériseront la poésie postsymbolique.

Cette situation peut être précisée, me semble-t-il, au niveau du contenu dans ses marques les plus évidentes: sa rhétorique explicite. Soit le deuxième vers :

2 Ils prolongent souvent des plaintes éternelles;

où des métaphores qui se doublent peuvent être réécrites de la manière suivante :

$2 a$ *ils miaulent souvent comme s'ils se plaignaient [...] = plaintes

$2 b \quad "[. .$.$] ces plaintes sont longues (durent) comme si on n'en voyait$ pas la fin = éternelles

Remarque: je rappelle que l'astérisque $\left(^{*}\right)$ précède conventionnellement une phrase (plus généralement une forme) qui n'a pas été réellement observée.

La première de ces métaphores (2a) appartient nettement au langage symboliste qui fait correspondre des domaines divers de l'univers, soit dans ce cas particulier le règne animal et la condition humaine: "miauler» et "se plaindre" déterminent des classes lexicales différentes, c'està-dire que leurs traits lexicaux (l'opposition sémique "animal »/ «humain ») devraient déterminer l'apparition de sèmes contextuels particuliers et qui devraient être redondants: ou bien "le chat miaule", ou bien "l'homme se plaint". Ici "le chat se plaint". La métaphore («le chat miaule comme s'il se plaignait») tend donc à organiser un univers symbolique de correspondances ("le chat miaule comme l'homme se plaint"), où le chat est implicitement le symbole de l'homme.

Remarque: Cette symbolisation, ici implicite, est souvent explicite dans l'œuvre de Nelligan, soit par exemple les vers des poèmes suivants (le titre en majuscules est précédé du numéro de la page où le poème se trouve dans les Poésies complètes):

165: LE SOULIER DE LA MURTE : "Mon âme est un soulier percé."

168: POTICHE : "Mon âme est une potiche où..."

183: LE LAC : “Ainsi la vie humaine est un grand lac qui..."

195: LA PASSANTE : "Ma jeunesse est pareille à la pauvre passante :..."

215: VIEUX PIANO : "O vieuX piano d'ébène, image de ma vie, / Comme toi..."

Les deux premiers vers du deuxième quatrains obéissent pour leur part à un autre sous-code rhétorique, le style artiste:

5 Parfois, leur dos aussi secoue un long frisson;

6 Leur poil vif se hérisse à des jets d'étincelles

Dans ce procédé rhétorique, le déterminant (généralement abstrait, puisqu'il est incident: ce peut être un adjectif) est mis ou pris pour le déterminé (généralement concret); 
par exemple :

La forêt sombre $\rightarrow$ Le sombre de la forêt

Remarque: Cet exemple est tiré d'un des Contes indiens de Mallarmé, "la Fausse Vieille"; voir ma Grammaire, op. cit., p. 219.

Ce procédé est aussi simple que répandu chez les prosateurs réalistes de la deuxième moitié du xixe siècle, mais son utilisation est complexe en ce qu'il peut s'effectuer à n'importe quel niveau de la constitution syntaxique. Alors que dans l'exemple que je viens de produire l'inversion se trouve au niveau du (et d'un seul) syntagme nominal, dans les deux vers des $\mathrm{CdN}$ le procédé se développe deux fois, d'abord au niveau de la phrase où le déterminant est le syntagme nominal et le déterminé le syntagme verbal :

$\mathrm{sn}]+\mathrm{sv} \rightarrow \mathrm{SN}_{1}(=\mathrm{sv})+\mathrm{SV}\left(=\mathrm{sn}_{1}\right)$

5 Leur dos/frissonne $\rightarrow$ * Un frisson/secoue leur dos

6 Leur poil/étincelle $\rightarrow$ * Des jets d'étincelles/hérissent leur poil

Remarque: La substitution des verbes “frissonner " et «étinceller " par "secouer" et "hérisser" apparaît comme la transformation de l'intransitif en un transitif dont le complément d'objet est une extension redondante du verbe (type : "La neige a neigé ») :

frissonner $\rightarrow$ faire/frissonner un frisson $\rightarrow$ secouer un frisson;

étinceler $\rightarrow$ faire/étinceler des étincelles $\rightarrow$ *hérisser des étincelles.

et ensuite au niveau des syntagmes nominaux sujets (déterminés) et objets (déterminants) :

$\mathrm{SN}_{2}+\mathrm{SV}+\mathrm{SN}_{1}$

5 Leur dos/secoue/un frisson

6 Leur poil/se hérisse/à des jets d'étincelles

bien que, dans cette deuxième application du précédé, seul le cinquième vers constitue une figure, puisque l'autre est en réalité le résultat d'une transformation passive (celle du verbe pronominal). Ce style artiste rejoint la métaphore symboliste dont il était question plus haut par l'inversion de l'abstrait au concret en même temps que du concret à l'abstrait: ainsi le poil et le dos du chat agissent ou subissent un patient ou un agent concrets et matériels symboliques d'abstractions immatérielles (le frissonnement, l'étincellement) et susceptibles dès lors d'organiser un univers symbolique de correspondances (frissonner et étinceler, comme se plaindre, non seulement de douleur ou de toute autre sensation physique, mais aussi de peur, d'angoisse ou de toute autre émotion humaine).

Remarque: le procédé est ici utilisé avec d'autant plus d'ingéniosité que les vocables "frissonner ", "se hérisser w et "étinceler " sont déjả susceptibles d'un transfert sémique du concret à l'abstrait (de la physiologie à la psychologie) largement grammaticalisé: "l'homme frissonne de peur" comme son regard "étincelle" de n'importe quel sentiment, comme encore il peut "se hérisser " devant n'importe quelle perception.

Quoi qu'il en soit, ces quatre traits de deux procédés rhétoriques (la métaphore et le style artiste) sont les seuls qui se trouvent explicite- 
ment dans ce texte. Ils sont assez nombreux pour assurer sa situation intertextuelle très nette (disons: pour préciser que ce texte est littéraire et qu'il appartient à ce domaine de la littérature - en particulier d'expression française - qu'on appelle la poésie symboliste de la fin du siècle dernier); mais le texte en est aussi assez sobre pour que soit maintenue au niveau du contenu l'unité harmonique qu'on a déjà décrite au plan de l'expression. Bref, les $\mathrm{CdN}$ se codifient nettement mais sagement comme une occurrence de la poésie symbolique: c'est déjà une grande sagesse pour une œuvre que de ne pas se laisser envahir par les codes ou les langages "littéraires", car elle conserve alors la liberté d'être l'essentiel - un texte, ce qui va nous occuper maintenant.

\section{Poétique : La signification poétique des CdN}

Un texte littéraire efficace conduit toujours, finalement, à la lecture poétique, celle que l'on reconnaît parfois dans le bavardage de l'histoire littéraire et presque toujours dans les études thématiques. Dans le cas du poème, il s'agit souvent de la seule information rationalisable ou en tout cas rationalisée. En voici d'abord un exemple très simple, mais qui me paraît bien significatif : à la lecture des $\mathrm{CdN}$,

Remarque : j'allais prendre une précaution, qui est significative parce qu'un langage poétique ne s'assure généralement pas dans un seul poème, en précisant: "à la lecture des Poésies de Nelligan..."; mais cette précaution est inutile, je pense, à la valeur démonstrative de mon exemple.

ne savez-vous pas, comme moi, que les chats dont il est question sont «noirs»? Et pourtant, sị vous relisez ce poème, vous remarquerez que cette «couleur» ne leur est jamais immédiatement attribuée. De la même manière, vous comprenez comme moi le sens poétique de ce texte même s'il est probable que vous serez insatisfaits de la description que je vais tenter d'en donner et qui pourrait amorcer n'importe quelle étude thématique: d'abord, l'homme, souvent, ne comprend pas pourquoi le chat miaule et encore moins pourquoi, en même temps, il écoute ces horloges de minuits que, par-dessus le marché, cet animal semble comprendre jusqu'à la peur (sinistre et affreux) et jusqu'à l'extase et à l'exaltation (mystique et solennel). Il s'agit, évidemment, d'un thème existentiel et fondamental qui met en scène l'esprit humain et qu'on peut traduire par la question suivante: "Ce que l'intelligence ou la conscience humaine ne peut sonder a-t-il tout de même un sens? "Question qui interroge aussi bien le matérialisme que l'existentialisme: existe-t-il de l'être hors de l'être ou de la conscience hors la conscience? Fondamentalement, les $\mathrm{CdN}$ me paraissent être cette interrogation du mysticisme et du fantastique. Ce texte transporte encore d'autres significations poétiques, mais qui se situent à mon sens à des plans moins fondamentaux que celuici (où la question reproduit la situation du lecteur qui cherche à "comprendre" les $\mathrm{CdN}$ ) : I'homme est bien comme le chat, incompréhensiblement attiré par cela même qui le repousse, l'angoisse et lui fait peur, et en 
particulier par le néant, ce qui n'existe pas (ce dont on ignore la raison), sinon par des chimères (comme ces horloges); mais le poète, surtout, est comme le chat que nous ne comprenons pas et qui se plaint en écoutant ces choses aussi horribles que merveilleuses; enfin, le chat est cet animal qui crie sans raison et qui cherche à comprendre ce qui en a encore moins. Dans tous les cas, on retrouve la fantastique mise en ceuvre du drame de la bête et de l'homme, du poète et de l'esprit: l'angoissante fascination pour leur impuissance (réelle ou supposée) devant l'extraordinaire ou le bizarre - la noirceur.

Bien entendu, je ne prétends pas épuiser par cette description la substance poétique des $\mathrm{CdN}$, mais on peut raisonnablement accepter qu'il s'agit là d'un bref résumé de son contenu poétique. On peut d'ailleurs en trouver une autre description dans la seule étude que je connaisse de ce poème, soit la situation que lui donne Paul Wyczynski dans l'univers imaginaire de la poésie de Nelligan. Ce critique situe les CdN dans le rêve vertical, c'est-à-dire le cauchemar qu'il oppose à la rêverie sous le nom de rêve horizontal, et qu'il appelle la "hantise du noir»; les chats prennent place avec les corbeaux, les perroquets et les hordes de bœufs qui figurent l'apparition des forces de la nuit bien lexicalisées par la Névrose et la Folie. Et je tiens, en effet, que les CdN exposent cet engouffrement du fantastique et du mysticisme dans l'univers; et que cet univers soit celui de la matière ou de la conscience, il est clair qu'il trouve là sa Folie. Dès lors, les connotations psychologiques et sociologiques apparaissent nettement : la destinée du poète, comme la société anachroniquement angélique où elle s'est déroulée, se correspondent trop bien pour qu'elles ne puissent pas être nettement désignées par cette Folie.

Bibliographie sommaire: Sur la lecture thématique, psychanalytique et socio-culturelle de l'ceuvre de Nelligan, voir respectivement :

Paul Wyczynski, Émile Nelligan, Sources et originalité de son œuvre, Ottawa, Éd. de l'Université d'Ottawa, 1960 (l'étudé des CdN, p. 226-227, est à comprendre dans sa situation dans tout le sixième chapitre: "Hantise du noir" ", p. 205-245) ;

Gérard Bessette, «Émile Nelligan», dans Une littérature en ébullition, Montréal, Éd. du Jour, 1968. ["Émile Nélligan »] : numéro spécial de la revue Etudes françaises, août 1967, vol. III, $n^{\circ} 3$, en particulier les “Documents" et les articles de Jean-Charles Bonenfant et de G.-André Vachon.

\begin{abstract}
Remarque : La classique et scolaire "explication de texte" se serait terminée par l'exposé du contenu thématique ou poétique des $\mathrm{CdN}$ qui vient d'être résumé : il s'agit en réalité d'un tour de passe-passe puisque tous les lecteurs connaissent ce sens d'avance et qu'on a beau jeu de le faire "apparaître "à la fin de l' "explication", comme si on le "découvrait " à ce moment. C'est donc bien volontairement que je l'expose avant les lectures stylistiques et sémiotiques: je voudrais éviter de me donner l'air de parvenir à ce que n'importe quel lecteur sait de science certaine - parce que le texte le dit clairement: un contenu thématique ou une information poétique connotée que j'ai simplement essayé de circonscrire de manière approchée et dont je crois avoir décrit ailleurs le mécanisme (voir la remarque de la p. 56).
\end{abstract}

Cette description du contenu poétique des $\mathrm{CdN}$ nous a conduits bien loin (puisqu'on en est tout simplement à «la Folie des univers de la matière 
et de la conscience»); pourtant, c'est finalement là qu'on en vient toujours avec n'importe quel texte littéraire et très immédiatement avec n'importe quel poème. En effet, dans le cas du texte narratif, par exemple, cette signification poétique (connotée) est toujours médiatisée par l'intermédiaire d'un contenu “immédiat", le contenu sémiotique (dénoté), qui est largement codifié sous le nom de contenu narratif. Par exemple, le thème de la "Nausée" chez Céline (voir l'article de Jean-Pierre Richard, "Nausée de Céline», N.R.F, août-septembre 1961) se dégage d'un traitement narratif de la signification (Bardamu en a effet, par exemple, la nausée à tel ou tel épisode du Voyage au bout de la nuit). Or c'est ce traitement de la signification qui fait tout de suite problème dans le cas d'un poème et en particulier ici, dans les CdN. Et c'est avec raison que Georges-André Vachon, par exemple, peut s'interroger de manière dramatique : «Qu'est-ce que cela veut dire?"

Remarque: G.-André Vachon, "Qu'est-ce que cela veut dire? " dans Variables de Michel Beaulieu, Montréal, Les Presses de l'Université de Montréal, 1973 , p. 93-99: c'est la question qu'il n'a cessé de poser, en particulier dans la revue Études françaises, par exemple entre son exposé sur cle Conflit des méthodes" (juin 1966, vol. II, $n^{\circ} 2$, p. 191-216) et sa "Note sur Réjean Ducharme et Paul-Marie Lapointe" (octobre 1975, vol. XI, n' 3-4, p. 355-387), s'interrogeant sur la signification de cet Hiver de force qu'on a tous mis quelques heures à lire comme allant de soi. Le texte qu'on lit actuellement doit être considéré comme ma réplique à et de la phrase suivante qu'on replacera dans le contexte du dernier article cité : "Un chat est un chat, une pomme une pomme; mais un texte n'est pas un texte." (p. 363).

En effet, si on juge acceptable la signification poétique des $\operatorname{CdN}$ telle que j'ai essayé plus haut de la circonscrire, je serais bien en peine de produire une telle saisie du sens immédiat de ce poème tel qu'il se donne dans une lecture qui s'interdit le déploiement thématique. Cela pourait donner: on ne comprend pas pourquoi les chats miaulent aux becs de gaz éteints et encore moins qu'ils frissonnent et se hérissent en entendant les horloges sonner minuit; d'autant qu'il apparaît bien plus normal que les chats frissonnent, se hérissent et écoutent lorsque les horloges sonnent qu'ils miaulent sans raison (alors que le texte inverse le développement, ce que j'ai indiqué par les mots de relation "encore moins"); d'autre part, le chat miaule, c'est le dictionnaire qui le dit, et il n'y a pas là de quoi fouetter un chat. Alors comment peut-on, sur le développement d'un sens immédiat pour le moins aussi pauvre, en arriver à une lecture poétique très riche? C'est la question à laquelle la sémiotique devra dans l'avenir apporter une réponse : je me contenterai de montrer comment le problème peut être posé dans le cas des CdN.

\section{Stylistique : La syntaxe des CdN}

Essayons de voir comment s'organise le contenu immédiat sur lequel se construit le contenu poétique que nous venons de résumer. La meilleure façon de décrire le plan de l'expression des CdN est d'en dresser la grammaire ou, en tout cas, la syntaxe sur la base de la gram- 
maire de la langue française qu'elle actualise. Les $\mathrm{CdN}$ se présentent, de ce point de vue, comme la suite de deux phrases, articulant respectivement deux et trois propositions, qui forment d'ailleurs chacun des deux quatrains, la phrase du premier quatrain articulant, selon la terminologie de la grammaire traditionnelle, une proposition subordonnée à une proposition principale; tandis que la phrase du deuxième quatrain concatène ou juxtapose deux propositions dont la première est une indépendante et la deuxième une principale, puisqu'elle articule une proposition subordonnée. C'est cette structure syntaxique de surface que suit rigoureusement la versification puisque chaque proposition constitue toujours un ou deux vers complets; or il est clair que la versification constitue une règle déterminante de la transformation des structures syntaxiques profondes en structures de surface telles qu'elles puissent la constituer. Par exemple, la différence entre les deux phrases suivantes :

$P_{1}$ : *Les becs de gaz qui sont éteints...

$P_{2}$ : Les becs de gaz éteints...

(c'est-à-dire entre l'application ou non de la transformation d'épithétisation qui se caractérise par l'effacement de la copule et du relatif), cette différence donc pourrait bien être plus indifférente dans un texte de prose que dans un texte versifié qui peut la déterminer; de sorte qu'il importe plus de décrire la structure profónde que la structure de surface qui peut se trouver largement déterminée par le code de la versification. Or ces deux phrases ou ces cinq propositions de surface peuvent être décrites comme la transformation de ce qui est, en structure profonde, six phrases minimales actives du type:

$\mathrm{SN}_{1}+\mathrm{GV}+\mathrm{SN}_{2}+(\mathrm{SPv})$

IIs/prolongent/des plaintes/aux becs de gaz;

Remarque: La phrase minimale est celle à laquelle on ne peut rien retrancher et si je l'appelle “active", c'est parce qu'elle peut connaître (ou non) une transformation passive, ce qui n'est pas le cas, par exemple, de la phrase attributive dont il sera question plus bas. SPv est le syntagme prépositionnel du verbe que la grammaire traditionnelle appelle le complément d'objet indirect: les parentheses indiquent qu'il est facultatif dans la grammaire de ce poème, comme les crochets indiqueront plus bas l'effacement.

II est d'abord remarquable que toute l'ossature syntaxique des CdN soit entièrement constituée par ce type de phrase, ce qui illustre encore à un autre niveau (j'y reviendrai) son caractère "harmonique" ou "monotone». A ces six phrases actives, s'ajoutent sept syntagmes prépositionnels compléments de la phrase $\left(S P_{p}\right)$ que la grammaire traditionnelle appelle les circonstants :

$\mathrm{P}_{1}+\left(\mathrm{SP}_{\mathrm{p}}{ }^{+}\right)$

Ils prolongent des plaintes aux becs de gaz/la nuit;

Où le signe + indique qu'ils peuvent se trouver dans chaque phrase en nombre variable: trois d'entre eux complètent la première phrase et deux 


\section{TABLEAU}

Stylistique : analyse syntaxique des $\mathrm{CdN}$

\section{1. ÉTAT DE LA STRUCTURE PROFONDE DES CdN}

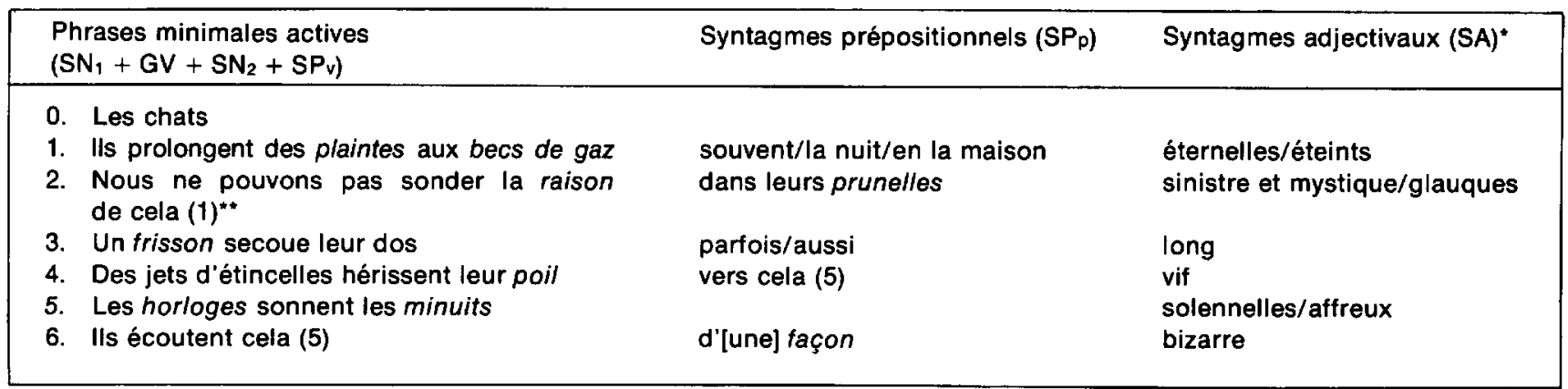

*Les adjectifs sont, dans l'ordre, les extensions des substantifs soulignés dans les phrases ou le SPp correspondants.

**Entre parenthèses, le numéro indique la phrase qui est pronominalisée. Je signale que dans le cas de cette deuxième phrase, je n'ai pas jugé utile de formaliser la négation et l'opérateur verbal (le semi-auxiliaire), mais on voit qu'il s'agit de la phrase "Nous sondons la raison de cela" à laquelle on applique la transformation négative et l'auxiliarisation de la possibilité (ce qui a pour effet de décupler la négation).

\section{LA SÉRIE de tRANSFORMATIONS APPLICABLES A CETTE STRUCTURE PROFONDE}

On peut supposer que les transformations nécessaires pour former les phrases de surface sont les suivantes (dans la mesure où je rationalise correctement l'inverse des opérations que j'ai effectuées pour en arriver à cet état de la structure profonde) où $\mathrm{Ph}$. signifie phrase et $\mathrm{p}$. proposition en surface :

Ph. 1, p. 1 : déplacement (des SP en tête de la phrase);

p. 2: coordination $(+E t)$; subordination avec lexicalisation de la négation (+ sans que) qui entraîne corrélativement le subjonctif (pouvons $\rightarrow$ puissions); pronominalisation du complément du nom (de cela $\rightarrow \theta n$ ); déplacement (du SP entre le verbe et son extension); 
Ph. 2, p. 1 : pseudo-transformation passive du style artiste (voir page 60); deplacement (ce qu'est essentiellement la transformation passive du style artiste (voir page 60); déplacement (ce qu'est essentiellement la transformation précédente; s'y ajoute celui des SP placés en tête de phrase et entre le sujet et le verbe):

p. 2 : transformation passive de la phrase (4); nominalisation de la phrase (5) : Les horloges sonnent les minuits $\rightarrow$ Les minuits des horloges qui sonnent;

p. 3: relativisation de la phrase (6) : Ils écoutent cela $(5) \rightarrow$ lls écoutent les minuits des horloges qui sonnent $\rightarrow$ les minuits des horloges (qui sonnent) qu'ils écoutent $\rightarrow$ suite de la nominalisation de la phrase (5) : les minuits d'horloges qu'ils écoutent sonner; déplacement : le seul déplacement porte ensuite sur l'adjectif “bizarre m.

\section{REMARQUE SUR LA SUBORDINATION}

Si la relativisation (intermédiaire dans le traitement de la phrase (5) ou essentielle dans le cas de la phrase (6) qui aboutit à la relative de surface $p$. 3) ne pose pas de problème, la "subordonnéen p. 2 est en réalité la proposition "principalen de la première phrase, ce qui explique qu'elle puisse être doublée d'un morphème de coordination. Maurice Grevisse (le Bon Usage, $\$ 1045,4^{\circ}$ ) décrit la proposition subordonnée circonstancielle introduite par asans que * (à laquelle il ne donne pas d'autre nom) en disant que ale mot sans sert alors à écarter l'idée de quelque fait accessoire m. II cite ailleurs ( $\$ 882$, b) l'exemple suivant tiré de Hugo: "La tête tourna sans que le corps ne remuât. "Sur cet exemple, on peut voir que les mots de relation sans que et le subjonctif marquent:

1. du point de vue du temps, la concomitance des deux propositions: "Quand la tête tourna, le corps ne remua pas»;

2. du point de vue de la relation, la restriction qui est constituée d'une opposition et d'une négation et qui est équivalente au mot de liaison (et non de subjonction) mais: "La tête tourna, mais le corps ne remua pas."

Or ces deux relations sont également contenues dans les mots de relation plus courants alors que :

Alors que la tête tourna, le corps ne remua pas:

Alors que les chats miaulent, nous ne pouvons pas en sonder la raison;

en quoi on voit d'abord que la principale est en réalité la subordonnée (et inversement) et ensuite que bien loin que cette proposition principale écarte “l'idée de quelque fait accessoire" elle est tout au contraire une mise en évidence (par le tour sans que + subjonctif) de la négation et de ce qu'elle frappe, ici le pouvoir d'en sonder la raison - la puissance de l'esprit.

\section{BIBLIOGRAPHIE SOMMAIRE}

Jean Dubois et Françoise Dubois-Charlier, Éléments de linguistique française: syntaxe, Paris, Larousse, “Langue et Langage ", 1970.

Jean Dubois, Grammaire structurale du français, 1: Noms et pronoms, II: le Verbe et III : la Phrase et les transformations, Paris, Larousse, "Langue et Langage ", 1965, 1967 et 1969.

Maurice Grevisse, le Bon Usage, Grammaire française, $8^{e}$ éd., 3e tirage, Paris et Gembloux, Hatier et Duculot, 1964. 
complètent la troisième; seule la cinquième phrase n'en comporte aucun, mais elle constitue elle-même un huitième syntagme prépositionnel. Enfin, on complète cette description syntaxique en disant que tous les syntagmes nominaux (SN) de ces phrases minimales, sauf évidemment dans les cas où ils sont constitués d'un pronom, et à deux exceptions près ( «dos" et «jets d'étincelles"), sont complétés par un syntagme adjectival (SA) formé d'un adjectif et dans un seul cas ( «raison") de deux, de sorte qu'on trouve en structure profonde neuf phrases attributives du type

$[\mathrm{SN}+$ être $]+(\mathrm{SA})$;

[ces plaintes sont] éternelles;

On trouvera le détail de cette analyse dans le tableau I: il illustre d'abord l'extrême simplicité syntaxique des CdN qui ne connaît qu'un type de phrase (la phrase minimale active) et que deux types d'extension (le syntagme prépositionnel et l'adjectif). On se convaincra aisément de cette simplicité en constatant que tous les verbes sont transitifs directs et qu'aucun n'est intransitif, impersonnel ou attributif, par exemple, ce qui compliquerait évidemment la structure de la phrase. Ce tableau a, d'autre part, l'avantage de séparer le noyau de l'information (les phrases minimales) de l'information périphérique (les syntagmes prépositionnels) et de l'information accessoire (les attributs ou les adjectifs) et de pouvoir constituer, éventuellement, une base d'analyse du contenu immédiat.

L'analyse qu'on trouve dans le tableau I fait seulement abstraction des ambiguïtés syntaxiques des $\mathrm{CdN}$ : deux d'entre elles, les plus importantes et qu'on repère à la première lecture, portent sur les mots de relation aux et vers et correspondent à leur emploi dans des tournures populaires, le premier cas, la préposition à peut introduire un complément d'objet indirect (SPv), comme j'ai choisi de l'analyser, ou un complément circonstanciel $\left(S P_{p}\right)$ tel qu'il se trouvait dans la version originale des $C d N$ qui s'ouvrait sur la participiale («les Becs de gaz éteints», Poésies complètes, p. 222) et qui peut marquer aussi bien le temps que la cause. En ce qui concerne la préposition vers, elle peut tout aussi bien marquer le temps comme dans la tournure populaire que la direction ou le lieu. Or il est remarquable que ces deux prépositions signifient l' "approximation" et qu'elles le sont, approximatives, par leur ambiguïté même; et qu'elles tournent en dérision le langage stéréotypé en le surchargeant de signification. Une troisième ambiguïté se trouve dans la situation d'un autre mot de relation, mais de type adverbial celui-là, le SP aussi qui peut être considéré comme une extension de la phrase (c'est ainsi qu'il est analysé dans le tableau I) ou seulement du verbe, de sorte qu'on peut lire ou bien "parfois également" ou "parfois en plus" (ce qui est le plus probable à cause de la fréquence du déplacement des SP dans les CdN), ou bien encore "leur dos pareillement et comme une autre ou n'importe quelle autre partie du corps secoue un long frisson». Toutefois, on doit remarquer que ces ambiguïtés ne peuvent pas avoir un grand impact sur la lecture en ce qu'elles n'accumulent pas des contenus très différents et certainement pas contradictoires. 
En bref, on peut dire que l'expression des $\mathrm{CdN}$ ou l'organisation syntaxique de ce texte confirme son caractère habituel. Je ne veux pas dire qu'on rencontre partout des textes qui organisent (ou s'organisent sur) des structures syntaxiques aussi simples; j'entends même exactement le contraire: il est bien possible qu'une phrase sur trois se construise en français sur le modèle de la phrase minimale active du type

$\mathrm{SN}_{1}+\mathrm{GV}+\mathrm{SN}_{2}$ : sujet, verbe et objet direct;

Remarque: il semble en effet qu'on puisse estimer à environ $30 \%$ le nombre de phrases françaises qui présentent dans un texte cette structure de surface, du moins dans le cas de la prose, ou bien scientifique (voir Jean-Claude Corbeil, Jes Structures syntaxiques du français moderne. Paris, Klincksieck, 1968, 1971, p. 73), ou bien narrative (Mallarmé, Grammaire générative des "Contes indiens", op. cit., p. 277); cette proportion pourrait bien n'être qu'un simple reflet de la fréquence relative de la phrase minimale active en structure profonde.

mais il faut bien admettre qu'il est inhabituel de trouver un texte qui répète cette structure six fois sur six; de la même façon qu'il faut se méfier de tout ce qui paraît trop «naturel ", il faut bien voir que l'habituel ne peut l'être «trop» sans artifice. Pourtant, il reste que la structure syntaxique des $\mathrm{CdN}$ ne peut que frapper par sa simplicité, sa monotonie et son harmonie, celles des structures courantes et redondantes que la structure de surface ne réussit pas à embrouiller. Or cette harmonie, bien propre à mettre en échec une théorie des écarts poétiques à l'emportepièce, a déjà été rencontrée jusqu'ici à tous les niveaux de notre description, depuis l'harmonie phonétique et rythmique de la versification, jusqu'à la sobriété manifeste du lexique, de la morphologie et de la syntaxe, sans compter la parcimonie des figures de rhétorique.

- Or justement, c'est ce caractère simple et habituel que renverse le contenu immédiat de ce texte. Mais une brève description sémiotique va nous montrer que cette simplicité se renverse, paradoxalement, simplement.

\section{Sémiotique : La signification immédiate des $\mathrm{CdN}$}

Considérons d'abord la structure temporelle des $\mathrm{CdN}$ qui pose et développe la règle de l'habituel. On remarque que le temps ou le tiroir verbal du texte est le présent marqué morphologiquement par tous les verbes à un mode personnel ("prolongent", "secoue», "se hérisse" et «écoutent»). II ne s'agit pas, néanmoins, d'un présent d'événement qui marquerait l'actualisation d'un fait à un moment donné du temps historique: bien au contraire, il s'agit du présent que les grammairiens appellent avec raison le "présent d'habitude", dont on peut produire l'exemple explicite suivant: "Je me lève tous les matins à sept heures" où le présent indique que j'en ai l'habitude. Et, comme dans cet exemple, un circonstant ( tous les matins") explicite généralement la fréquence de l'habitude; dans les CdN, il s'agit de l'adverbe "souvent " qu'on trouve 
au deuxième vers, de même que l'adverbe «parfois» qui ouvre le deuxième quatrain. On remarque en effet que le présent d'habitude, qui marque toujours une discontinuité temporelle par rapport au présent atemporel ou éternel, mesure de façon différente la fréquence d'apparition de l'habitude dans chacune des deux phrases du texte qu'opposent les deux syntagmes prépositionnels marquant le temps (souvent/parfois) et que confronte un troisième syntagme prépositionnel, l'adverbe aussi. On constate pourtant que cette opposition n'est pas catégorique (contrairement, par exemple, à l'opposition toujours/jamais) et qu'au contraire ces deux adverbes différencient un contenu sémique très mince qui peut être décrit comme deux orientations opposées (vers la fréquence et la rareté) à partir d'une même position (le sème «intermittence" ou "discontinu" qui leur est essentiel), comme l'illustre la figure 2. Or, comme pour mieux marquer l'habitude, c'est dans cette forme temporelle de l'intermittence ou du discontinu que vont se situer des contenus paradoxalement continus (et c'est à cause de ce paradoxe que ces derniers apparaîtront comme des figures ou des écarts, ainsi qu'on l'a vu dans un cas dès le début). En effet, c'est souvent (“discontinu») que les chats prolongent (faire durer $=$ "continu $"$ ) des plaintes (comme le miaulement, cri qui dure $=$ «continu») éternelles ( continu»); de la même façon que parfois ("discontinu») leur dos secoue un long («continu») frisson (qui comprend le sème contradictoire "momentané " mettant en relief le sème “continu» de l'adjectif). La figure des horloges résume ce traitement du temps dans les $\mathrm{CdN}$ : les horloges marquent toujours le temps ( continu») mais ne le manifestent qu'aux minuits («discontinu») et elles le font de manière solennelle (longuement? « continu »?).

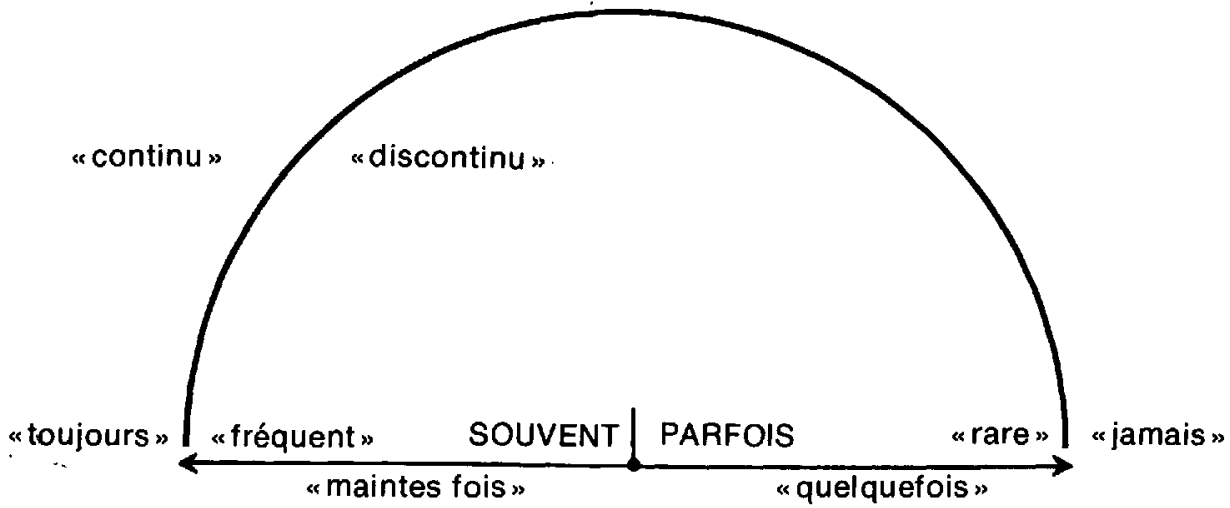

Figure 2. Analyse des orientations contraires de la position de discontinuité représentée par les deux adverbes «souvent » et «parfois».

Ce temps de l'habitude, ce discontinu habituel, est encore marqué explicitement par d'autres syntagmes prépositionnels : "la nuit", dont le déterminant singulier indique bien le discontinu (chaque ou toutes les 
nuits) et «Les minuits" où le déterminant au contraire pluriel marque encore le même contenu. Mais ce temps est aussi le lieu de l'habitude: "la nuit, en la maison", de sorte que s'applique à l'espace le discontinu de la structure temporelle. Bref, l'information périphérique a pour fonction de situer l'événement ou le noyau de l'information comme l'habituel, ce qui se produit habituellement - souvent et parfois - la nuit, en la maison. Cet événement habituel (le noyau de l'information), nous le connaissons comme celui qui se développe dans les six phrases minimales actives que l'on a rangées en tête du tableau I: les chats prolongent incompréhensiblement des plaintes, frissonnent, étincellent et écoutent sonner les minuits. Or, ce qu'on a appelé déjà l'information accessoire, et cela me paraît remarquable, va présenter comme l'inhabituel absolu ce qui vient justement d'être situé comme se produisant habituellement.

Deux paires d'adjectifs se présentent explicitement en opposition catégorique dans la structure de surface: premièrement, c'est évident, "sinistre»/ «mystique" qui se rapportent ensemble à la raison inexplicable de la plainte des chats; ensuite, de manière moins nette peut-être mais qui présente le même type d'opposition catégorique, «affreux»/ "solennelles» qui se rapportent tous deux aux minuits d'horloges, le premier qualifiant le déterminé et le deuxième le déterminant, déterminé et déterminant qui forment ensemble le syntagme nominal pronominalisé par le relatif "que": en effet, ce ne sont ni les minuits, ni les horloges que les chats écoutent, mais bien les minuits d'horloges, ce qui illustre bien la solidarité des deux adjectifs contradictoires. Cependant, dans chacune de ces séries, les adjectifs ne «s'opposent" pas de manière aussi immédiate que pourrait le laisser croire la structure de surface: pour le voir, il suffit de reconstituer la structure profonde de la signification telle qu'elle apparaît lorsqu'on prend en considération la structure sémique globale et les transformations sémantiques que supposent non seulement la structure de surface, mais aussi la structure syntaxique profonde qui se trouve présentée dans le tableau I. En effet, si l'on jette un coup d'œil sur le tableau II, on verra que l'on peut essayer de décrire la structure sémiotique des $\mathrm{CdN}$ de la même manière qu'on a déjà décrit sa structure syntaxique. A partir d'un petit nombre de règles simples (qu'on trouvera exposé dans ce tableau), la négation, la contradiction, l'intensification et la synthétisation, on peut décrire de manière efficace et économique la valeur sémiotique des éléments lexicaux (les lexèmes) utilisés pour construire les phrases de la structure profonde consignée en tête du tableau I. Ainsi, on voit que non seulement l'essentiel de la structure sémique de l'information accessoire (les SA), mais le sujet même du texte (du titre au sujet réellement pronominalisé) est le résultat de la négation de l'habituel ou du familier qui serait représenté par les chats familiers ou domestiques, ceux qui ronronnent. En effet, le sujet des CdN est constitué par ce que les chats ont de plus «sauvages": ce sont ceux qui crient (qu'ils miaulent ou qu'ils se plaignent), qui frissonnent et étincellent et, par-dessus tout, ceux qui écoutent (d'autant plus qu'ils miaulent et écoutent). Cette négation fon- 


\section{TABLEAU II}

Sémiotique : analyse de la structure sémique des CdN

\section{1. ÉtAT de LA STRUCTURE PROFONDE DES CdN}

a) Structure sémique du familier dans le lexème "chat"

\begin{tabular}{|l|l|l|l|}
\hline \multicolumn{2}{|c|}{ Opérateurs sémiques : } & positif & négatif \\
\hline \multirow{2}{*}{ 1. L'être } & 1.1 attribut & domestique (familier) & sauvage (non familier) \\
\cline { 2 - 4 } & 1.2 manifestation & ronronnement & miaulement \\
\hline \multirow{2}{*}{ 2. Le faire } & 2.1 action & $\begin{array}{l}\text { frileux (craint et } \\
\text { évite le froid) }\end{array}$ & $\begin{array}{l}\text { frissonner } \\
\text { se hérisser } \\
\text { étinceler }\end{array}$ \\
\cline { 2 - 4 } & 2.2 réaction & le sommeil & l'écoute \\
\hline
\end{tabular}

b) Structure sémique du familier dans les SA en opposition

\begin{tabular}{|l|l|l|l|l|l|}
\hline \multirow{2}{*}{$\begin{array}{l}\text { Opérateurs } \\
\text { sémiques }\end{array}$} & \multicolumn{2}{|c|}{ atténuatif } & \multicolumn{2}{c|}{ intensif } & \multirow{2}{*}{$\begin{array}{l}\text { opérateurs } \\
\text { sémiques }\end{array}$} \\
\cline { 2 - 6 } & positif & négatif & positif & négatif & favorable \\
\hline FAMILIER & CONNU & MYSTÉRIEUX & ORDINAIRE & GRAVE & défavorable \\
\hline HABITUEL & RASSURANT & MENACANT & TRANQUILLISANT & EFFRAYANT \\
\hline
\end{tabular}

où les sèmes retenus sont inclus dans les lexèmes de la manière suivante :
1. "mystérieux" $\rightarrow$ MYSTIQUE
2. "menaçant " $\rightarrow$ SINISTRE
3. "grave $\rightarrow \rightarrow$ SOLENNEL
4. "effrayant " $\rightarrow$ AFFREUX 


\section{LA SÉRIE de TRANSFORMATIONS APPLICABLES A CETTE STRUCTURE PROFONDE}

1. La négation: à tous les niveaux, c'est toujours l'élément négatif de la dichotomie sémique positif/négatif qui est choisi, par exemple, pour les chats domestiques/non domestiques, la raison connue/mystérieuse ou rassurante/menaçante.

2. La contradiction: dans le cas de la structure sémique des SA, deux sèmes (négatifs) contradictoires sont retenus en même temps, un sème favorable et un sème défavorable; mais qu'ils solent favorables ou défavorables, les sèmes retenus ont en commun le fait d'être tous négatifs.

3. L'intensification: ces deux transformations (la négation et la contradiction) s'intensifient progressivement: sinistre/ mystique $\rightarrow$ affreux/solennel. Cette intensification ne s'applique pas seulement à la structure sémique en cause ici : elle décrit également le passage de l'«inexplicable» à l' cinexpliqué ": la négation de l'auxiliaire de possibilité (marqué en plus du subjonctif : "puissions") s'appliquant au groupe verbal "sonder la raison" entraîne très nettement le sème "inexplicable», tandis que le développement de la description des chats et surtout l'adjectif BIZARRE impliquent au contraire le sème “inexpliqué».

4. La synthétisation de cette dialectique, sa pétrification, est encore contenue dans le lexème BIZARRE, c'est le sème «extraordinaire ", négation et contradiction (et leur intensification finale) du familier et de l'habituel. 
damentale sera reprise à tous les niveaux, et jusqu'à la fin, sur tous les éléments marquant le familier ou l'habituel, aussi bien d'un point de vue favorable que défavorable. Le tableau II illustre comment se développent la négation, la contradiction et l'intensification du familier et de l'habituel, bref le développement de l'extraordinaire, extraordinaire qui peut bien être favorable ou défavorable (c'est la contradiction), mais qui est forcément négatif (c'est la négation).

Voilà ce qui rend compte du fait que les chats soient "noirs"! En effet, de la même manière que l'intensification nous fait passer du rassurant "inexplicable» au menaçant “inexpliqué» (voir la troisième transformation du tableau II), les CdN épaississent constamment l'obscurité par l'intensification de la lumière. Les syntagmes prépositionnels de la phrase (1) (tableau I) situe non seulement cette phrase, mais tout l'événement, dans le temps et l'espace de l'obscurité: c'est la nuit (intensification: "les minuits") dans la maison où les becs de gaz sont éteints; or l'intensification de l'obscurité ne va pas consister à l'attribuer aux chats (les chats, le dos ou le poil "noirs»), mais à leur attribuer au contraire une lumière éclatante ( glauques prunelles ", intensification : “ jets d'étincelles "), de sorte que les chats produisent par contraste l'obscurité - leur propre noirceur.

Je pense que ce traitement de la signification immédiate des CdN tel que je viens de le décrire brièvement est propre à rendre compte de la signification poétique que j'ai essayé de circonscrire au début de cette analyse. On voit bien maintenant en quoi les CdN sont «noirs", et comment ce texte met en scène, dans le cadre le plus habituel et le plus familier (et cela de la versification au traitement de l'information périphérique en passant par la syntaxe), l'apparition de l'inhabituel et du non-familier, de l'extraordinaire et du fantastique - bref, l'intrusion de la Folie, marquée aussi bien dans la versification par les hiatus que par le traitement de la structure sémique de l'habituel produisant cet “habituel non-familier» qu'est la Névrose. L'Extraordinaire.

Et je précise encore que rien de tout cela ne constitue une “explication ", ni des $\mathrm{CdN}$, ni de leur signification poétique : il s'agit seulement d'un essai de description, que j'espère en conséquence aussi discutable que périmable.

\section{Quelques dimensions littéraires des $\mathrm{CdN}$}

Je voudrais revenir, pour conclure, à la littérature dont le texte qui vient d'être décrit est une occurrence. Comme mon travail n'est pas terminé, je l'ai dit dès le début, je me contenterai de livrer quelques notes fragmentaires pour illustrer la situation des $\mathrm{CdN}$ dans l'univers de la littérature. 


\title{
5.1 Le sonnet inachevé
}

Du point de vue stylistique, tout le monde vous le dira, les CdN sont un «sonnet incomplet».

\begin{abstract}
Remarque : "Le manuscrit de la collection Nelligan-Corbeil, d'un seul feuillet, n'a que deux quatrains de ce qui devait être un sonnet " (Luc Lacourcière, Poésies completes, op. cit., p. 322). Paul Wyczynski est encore plus affirmatif: ["Les chats» sont] deux quatrains d'un sonnet inachevé à moins que la page suivante, contenant les deux tercets, ait été perdue" (Bibliographie descriptive, op. cit., p. 28) et il avait déjà dit qu'il s'agissait d'un "sonnet inachevé conçu au printemps de 1898 " (Émile Nelligan, Sources et originalité de son cuure, p. 303 , note 7), sans dire comment il parvenait à cette belle conclusion.
\end{abstract}

Or rien, absolument rien n'indique qu'il pourrait s'agir d'un poème inachevé. Mais je pense que c'est la Littérature qui a imposé cette idée folle à la critique: l'œuvre de Nelligan d'abord (près de la moitié des poèmes des Poésies sont des sonnets: Paul Wyczynski en compte 74 sur 168 poèmes, Sources et originalité, p. 298) et ses «sources" ensuite ("les Chats" de Baudelaire et "Mon chat" de Jules Lemaître), étant bien entendu que si Nelligan s'inspire d'un sonnet, il ne vas pas inventer un poème de deux quatrains! Pourtant le même Paul Wyczynski compte 73 poèmes strophiques : est-ce que chaque fois qu'ils auront moins de trois strophes il s'agira de «poèmes inachevés"? Pourquoi, par exemple, "le Berceau de la Muse " (Poésies complètes, p. 62), qui se compose de deux tercets sur trois rimes, n'est-il pas, lui aussi, un "sonnet inachevée"? Parce qu'on ne commence pas un sonnet par la fin? Enfin, "Cœurs blasés" (Poésies complètes, p. 211) est encore un "sonnet inachevé" pour Paul Wyczynski (Sources et originalité, p. 303, note 7)... mais celui-là, il a été publié par le poète le 22 août 1896 dans le Samedi (p. 6)! En quoi on voit que la Littérature nous impose des idées en dépit des textes: il s'agit bien d'une institution sociale et non d'une simple suite de textes. On peut en tout cas se demander ce qu'il faudrait ajouter aux $\mathrm{CdN}$ pour en faire un poème complet. Réponse : deux tercets.

\subsection{Les CdN dans l'œuvre}

Du point de vue thématique, la situation des $\mathrm{CdN}$ dans les Poésies complètes est tout à fait remarquable: ce texte ouvre une dernière série (de six poèmes) des "Poèmes posthumes" que Luc Lacourcière qualifie très justement de "fantastique comme le sont par exemple les contes d'Edgar Poe». Il est heureux, je pense, que les Poésies complètes commencent à se fermer sur ce texte obscurcissant. II faut en rendre hommage à leur éditeur, Luc Lacourcière, comme il faut signaler sur ce point l'admirable analyse de Paul Wyczynski qui, on l'a vu, décrivait correctement cette “Hantise du noir".

\subsection{Les CdN dans le symbolisme romantique}

Du point de vue sémiotique, enfin, on doit signaler le renversement qui s'opère dans les CdN à partir des «Chats» de Baudelaire, chats fami- 
liers, aussi frileux que rassurants, ainsi que “Mon chat " de Jules Lemaître (encore plus familier, comme l'indique le déterminant dès le titre). Alors que Paul Wyczynski signale ces ressemblances, particulièrement avec le dernier tercet du sonnet de Baudelaire dont les $\mathrm{CdN}$ constituent la réécriture,

Leurs reins féconds sont pleins d'étincelles magiques,

Et des parcelles d'or, ainsi qu'un sable fin,

Étoilent vaguement leurs prunelles mystiques.

ce sont au contraire les différences radicales qu'il faudrait remarquer. Contrairement au texte de Nelligan, la syntaxe du sonnet de Baudelaire est complexe et variée, "inhabituelle", tandis qu'au contraire son contenu est «positif» (voir le tableau II) et il ignore la contradiction.

Par contre, le rapport que signale aussi Paul Wyczynski et auquel fait allusion Luc Lacourcière avec «le Chat noir » d'Edgar Poe est patent : c'est le même fantastique, la même terreur et la même folie s'intensifiant tout au long du conte de Poe qui se termine où les $\mathrm{CdN}$ commencent, sur le cri du chat :

Une plainte, d'abord voilée et entrecoupée, comme le sanglotement d'un enfant, puis, bientôt, s'enflant en un cri prolongé, sonore et continu, tout à fait anormal et antihumain, - un hurlement, - un glapissement, moitié horreur et moitié triomphe, - comme il en peut monter seulement de l'Enfer, - affreuse harmonie jaillissant à la fois de la gorge des damnés dans leurs tortures, et des démons exultant dans la damnation.

(Nouvelles Histoires extraordinaires, trad. de Charles Baudelaire, éd. du Livre de poche, 1964, p. 24.)

Bref, un miaulement, celui des CdN.

Guy Laflèche, Université de Montréal. 